\title{
Update on the treatment of systemic sclerosis
}

\author{
Arturo Rodríguez-de-la-Serna ${ }^{1,2, *}$ \\ ${ }^{1}$ Hospital de la Santa Creu i Sant Pau, Universidad Autónoma de Barcelona; ${ }^{2}$ Práctica privada de Reumatología. Barcelona, Spain
}

\begin{abstract}
Progressive systemic sclerosis, also known as scleroderma, is a rheumatic disease of autoimmune origin in which the main affectation occurs at the level of the arterioles and microvasculature, progressing toward fibrosis and vascular obliteration. At a skin level, a pathological increase in fibrosis causes the skin to harden and it also affects the subcutaneous cellular tissue, with the disappearance of the annex (hence, the name of scleroderma). In addition, there is an alteration of different internal organs, especially the lung and kidney, which gives it a poor prognosis although joint structures in the digestive system are also affected. The present article is an update of the current treatments for each affect, and of some up-to-date investigations that are improving the results and the prognosis. At present, there are different dilator drugs for treating Raynaud's phenomenon, such as calcium channel blockers, prostacyclin analogs, and phosphodiesterase-5 inhibitors, among others. Immunosuppressants, such as mycophenolate and others, are used for cutaneous sclerosis. For pulmonary involvement in the form of interstitial lung disease, immunosuppressants such as cyclophosphamide, mycophenolate, and biological treatments such as nintedanib or rituximab (RTX) are used. Pulmonary hypertension is managed with endothelin receptor antagonists, such as bosentan, phosphodiesterase inhibitors, or prostacyclin. The prognosis for scleroderma renal crisis has drastically changed since the initiation of treatment with angiotensin-converting enzyme inhibitors. Finally, bone marrow, lung, and heart transplants are used in extreme cases.
\end{abstract}

Keywords: Progressive systemic sclerosis. Interstitial lung disease. Pulmonary hypertension. Scleroderma renal crisis. Raynaud's phenomenon.

\section{Introduction}

Progressive systemic sclerosis (PSS), also known as scleroderma, is a rheumatic disease of autoimmune origin, in which the main affect is at the arterioles and microvasculature, with progression toward fibrosis and vascular obliteration. There are two main forms of phenotypic involvement: a diffuse form that affects the skin of practically the entire organism, including the skin of the face, torso and extremities, and a localized form, which only affects the extremities and does not extend above the elbows or knees.

In $10 \%$ of people who suffer from the disease the skin is not affected, this modality being called PSS sine scleroderma. The prognosis of the disease depends fundamentally on the existence of visceral involvement and, within it, of the lung, in the form of interstitial lung disease (ILD) that usually presents as a serious form. Pulmonary arterial hypertension (PAH) and primary heart damage are also present, these visceral forms being the cause of the high mortality of the disease. This is considered according to the latest study published, in which mortality increased from 1968 to 2000 in the United States, but since then there has been a decrease between 2001 and 2015. However, when compared with other causes of death, mortality remains high. In addition, it presents a disproportionate change between gender and race after observing it for 48 years, calculating
Correspondence:

*Arturo Rodríguez-de-la-Serna

E-mail: arturojj@ hotmail.com
Available online: 25-01-2022

J IMIDs. 2022;2(1):20-28

www.JournalofIMIDs.com
Accepted: 09-11-2021

DOI: 10.24875/JIMIDS.M21000014

rg/licenses/by-nc-nd/4.0/). 
an age-standardized mortality rate (ASMR) for SSc and non-SSc (all other causes), women and African-American persons had higher SSc ASMRs to non-SSc ASMR ratios than men and Eurasian persons, respectively".

In less than $5 \%$ of those affected, renal impairment occurs in the form of a renal crisis, which also carries a poor prognosis ${ }^{2}$.

Skin involvement serves as a prognostic marker in ESP, with diffuse forms having the worst progression ${ }^{3}$.

In recent years, the use of bone marrow transplantation, as well as lung transplantation, has been a valid alternative for some patients. It is also possible that stem cell transplantation will be a therapeutic alternative for these patients in the future 4 .

To address the treatment, the clinical manifestations in scleroderma are (Table 1):

- Raynaud's phenomenon (RP), digital ulcers and sclerosis of the skin, often accompanied by telangiectasia (Figure 1) and in the most advanced forms, leads to limited mobility, even opening the mouth (Figure 2)

- Scleroderma renal crisis

- PAH

- ILD

- Alteration of the gastrointestinal tract

- Heart disease

- Arthritis

The objectives of treatment are:

- Limiting or stopping progression of the disease

- Reducing sequelae

- Improving quality of life by managing disability and functional limitation.

\section{Treatment for Raynaud's phenomenon (RP)}

The treatment for RP depends on its severity. The objectives are to reduce the number and severity of crises, to improve the quality of life, and to avoid structural damage.

There are a series of vasoconstrictor drugs that should be avoided or used with great caution in the presence of RP:

- Nasal decongestants for local or general use, such as ephedrine compounds

- Anti-migraine ergotamine derivatives

- Beta-blockers, including glaucoma drops

- Drugs for hyperprolactinemia.

- Anti-Parkinson drugs.

In the treatment itself, it is important to consider some non-pharmacological measures such as protection against
Table 1. Systemic sclerosis treatment according to clinical manifestation

\begin{tabular}{|c|c|}
\hline Manifestation & Treatment \\
\hline \multirow[t]{4}{*}{ RF } & Calcium channel blockers \\
\hline & Prostacyclin analogs \\
\hline & Endothelin antagonist $A$ and $B$ \\
\hline & Phosphodiesterase- 5 inhibitors \\
\hline Digital ulcers & Endothelin receptor antagonist \\
\hline \multirow[t]{3}{*}{ Cutaneous sclerosis } & MTX \\
\hline & MMF \\
\hline & Cyclophosphamide \\
\hline \multirow{2}{*}{$\begin{array}{l}\text { Locomotive apparatus } \\
\text { damage }\end{array}$} & NSAIDs \\
\hline & Corticosteroids \\
\hline \multirow[t]{7}{*}{ ILD } & Oxygen therapy \\
\hline & Cyclophosphamide \\
\hline & MMF \\
\hline & Corticosteroids \\
\hline & Rituximab \\
\hline & Tocilizumab \\
\hline & Nintedanib \\
\hline \multirow[t]{5}{*}{$\mathrm{PH}$} & Endothelin receptors antagonists \\
\hline & Phosphodiesterase- 5 inhibitors \\
\hline & Prostacyclin \\
\hline & Prostacyclin receptor agonists \\
\hline & Lung and heart transplant \\
\hline \multirow[t]{2}{*}{ CRS } & ACE inhibitors \\
\hline & Alpha-adrenergic blockers \\
\hline \multirow[t]{3}{*}{ Digestive disorders } & Proton-pump inhibitors \\
\hline & Prokinetic treatment \\
\hline & Antibiotics \\
\hline
\end{tabular}

FR: Raynaud's phenomenon; ILD: interstitial lung disease; PH: pulmonary hypertension; CRC: renal crisis; MTX: methotrexate; MMF: mycophenolate m; NSAIDs: nonsteroidal anti-inflammatory drugs.

cold and micro-trauma, and avoiding tobacco. Alternative treatments such as biofeedback techniques have not been sufficiently studied from a scientific point of view.

\section{Pharmacotherapy}

1. Calcium channel blockers (CCB). They are the first line treatment, including nifedepine, diltiazem, 


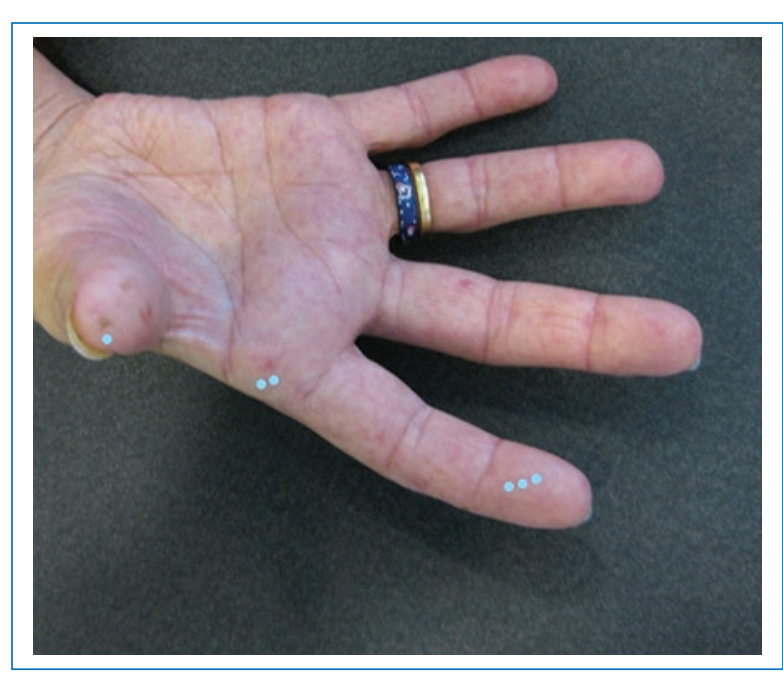

Figure 1. Patient's hand with scleroderma in the edematous phase in which the following can be observed.

*Digital ulcer on the thumb pad.

**Telangiectasias in the palm of the hand.

***Raynaud's phenomenon on the fingertips.

nicardipine, nimodipine, amlodipine and felodipine. They are effective in reducing both the number and severity of RP seizures. Its effect varies with dose, and higher doses tend to show greater efficacy. An example would be nifedepine, at doses of $30 \mathrm{mg} /$ day with a $30 \%$ reduction in seizures ${ }^{5}$.

2. Prostacyclin analogs. lloprost administered intravenously at doses of $0.05 \mathrm{mg}$ each day, has shown efficacy in the treatment for RP secondary to scleroderma by reducing the severity and frequency of attacks and as prevention for digital ulcers. Oral iloprost appears to be less effective ${ }^{6}$.

3.1-5-phosphodiesterase inhibitors. Sildenafil, tadalafil, and vardenafil have been used as an alternative in the case of resistance or intolerance to CCBs. They have shown limited efficacy.

4. Angiotensin blockers. Losartan at single doses of $50 \mathrm{mg} /$ day has been proposed as compassionate use in the treatment for RP in cases of intolerance to calcium channel inhibitors.

5. Angiotesin converting enzyme inhibitors. Captopril and enalapril at doses of $20 \mathrm{mg} /$ day have been used as compassionate use, in case of coexistence with $\mathrm{PAH}^{7}$.

6. Serotonin receptor antagonists. Fluoxetine at doses of $20 \mathrm{mg} / \mathrm{day}$ is proposed for compassionate use, based on a small, randomized study, which showed it to be somewhat superior to nifedepine ${ }^{8}$.

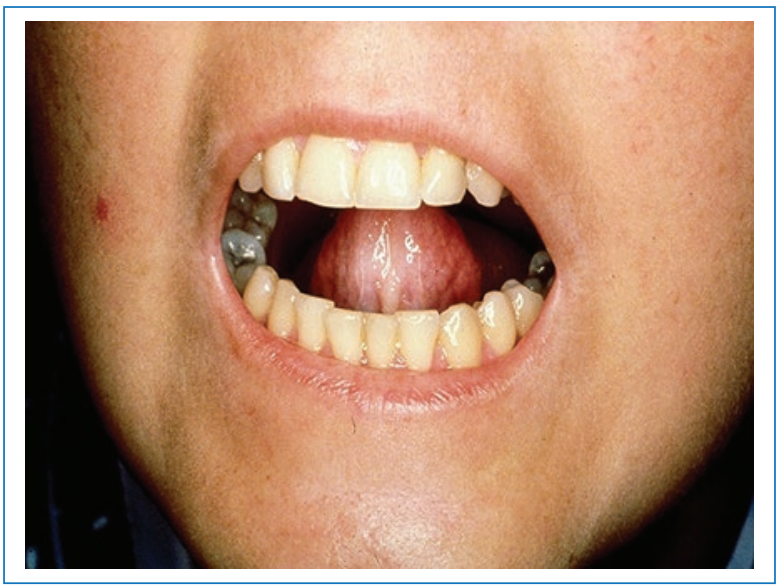

Figure 2. Patient with advanced systemic sclerosis, with generalized skin involvement, who had difficulty opening the mouth that can be measured, and even evaluated, by the distance in $\mathrm{cm}$ between both lips or between the upper and lower incisors.

\section{Treatment of digital ulcers}

The treatment of digital ulcers, in addition to local care and the prevention or treatment of infections when they occur, does not differ from the treatment of RP, with which they are also usually associated. Endothelin receptor antagonists such as bosentan have proven to be effective in preventing ulcers, but they have not shown an effect on the on the speed of healing ischemic wounds. There is no indication on prescribing bosentan as a curative treatment for digital ulcers ${ }^{9}$. The use of cellular therapy techniques by digital injection of stromal vascular fraction or mesenchymal stem cells is still under investigation. These techniques are, therefore, not currently recommended ${ }^{10}$.

\section{Treatment for cutaneous sclerosis}

There is no approved treatment for treating skin sclerosis specifically. Different immunosuppressants are used based on the autoimmune pathogenesis of the disease and some inconclusive clinical trials.

\section{Methotrexate}

There are some trials that showed improvement in scleroderma of short evolution ( $<3$ years), without statistical significance, and it is used on the recommendation of expert groups and therapeutic guidelines at weekly, oral or subcutaneous doses of $0.3 \mathrm{mg} / \mathrm{kg}$, and for a period of 2 years. 


\section{Cyclophosphamide}

Although there are different trials for its use in pulmonary involvement, there are limited and inconclusive experiences for skin involvement. The SLS-I study evaluated oral cyclophosphamide and showed a discrete improvement at the cutaneous level, which was significant after one year of treatment, and which disappeared after discontinuation of treatment ${ }^{11}$.

\section{Mycophenolate MOFETIL (MMF)}

There is no direct study on the use of MMF as disease-modifying anti-rheumatic drugs (DMARD) for SSc, but there are observational and also controlled studies for lung damage. In the SLS-II trial, MMF use was associated with a 4.9-point reduction in the modified Rodnan score versus 5.3 for cyclophosphamide at 24 months. The results are only significant in diffuse forms of SSc. Analysis of SLS-II compared with the SLS-I placebo group would suggest that MMF use was associated with an improvement of the modified Rodnan score compared with the placebo group after 24 months. Considering these data, it is considered to use MMF DMARD in the diffuse cutaneous forms of SSc with or without lung damage. The recommended dosage of MMF is from 2 to $3 \mathrm{~g} /$ day.

The skin must be treated locally for good hydration. It is recommended to use moisturizing and softening creams and lotions several times a day. Paraffin baths for the hands or the use of castor oil have not been rigorously and scientifically studied. Personalized physiotherapy with massages aimed at softening the skin or subcutaneous tissues can be proposed, although no rigorous study on the subject has been conducted to date.

\section{Treatment of locomotive apparatus damage}

Locomotive apparatus damage is frequent with SSc: arthralgia, arthritis, and tenosynovitis are especially common in the $1^{\text {st }}$ years of the disease. Fibrous tenosynovitis is included in the disease activity score and is considered a sign of worsening development. No randomized study has specifically addressed musculoskeletal damage ${ }^{12}$.

- Arthralgia and arthritis can be treated by analgesics and by nonsteroidal anti-inflammatory drugs in the short term, with monitoring of renal function and after evaluation of digestive bleeding risk.

- Oral corticosteroids are commonly proposed at an initial dosage of prednisone equivalent to but not

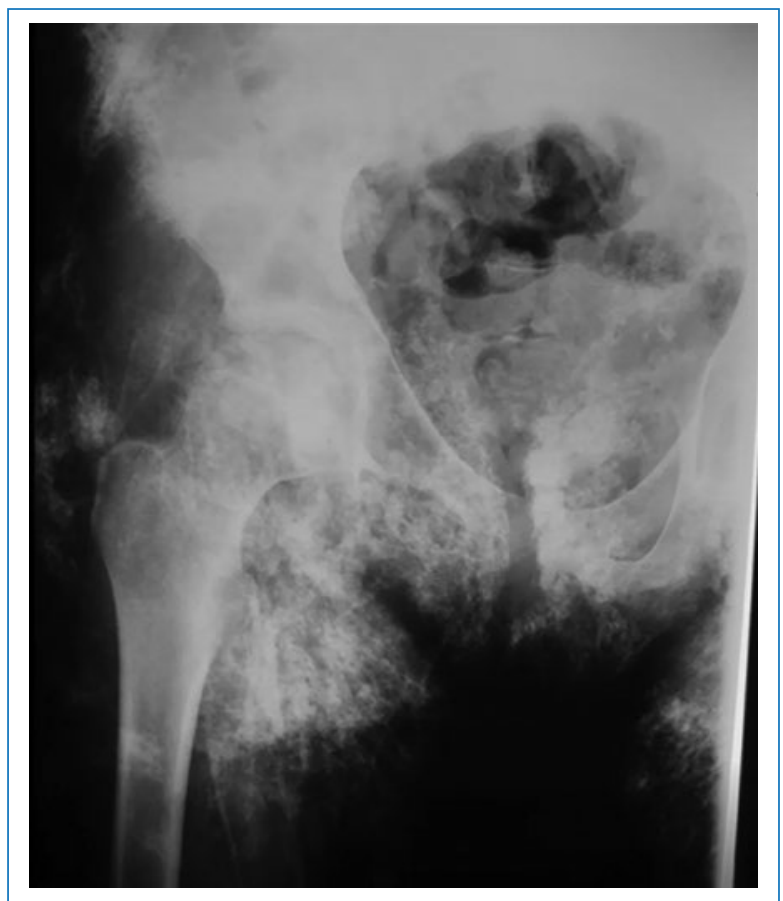

Figure 3. Pelvic $\mathrm{X}$-ray, where multiple dystrophic calcifications are observed.

exceeding 10-15 mg/day, then at a lower long-term dosage of less than $10 \mathrm{mg} /$ day.

- Corticosteroid shots can be proposed in case of articular or tenosynovial damage.

- Tenosynovitis can benefit from the same treatments as articular damage, but there has not been any specific trial dedicated to this type of damage.

- Rehabilitation programs can reduce disability, but they have not been shown to have significant long-term efficacy.

- Calcinosis: the frequency of this complication is high (near $25 \%$ of all patients) and is the source of pain and disability (Figure 3). No treatment has been shown to be effective. In certain situations, surgical excision of calcium deposits can be proposed to promote healing and avoid secondary infections after making sure that the peripheral vascular condition allows for it.

\section{Interstitial lung disease treatment}

- Total and definitive smoking cessation.

- It is recommended to give an annual flu vaccination and an anti-pneumococcal vaccination.

- Oxygen therapy: as with other causes of chronic respiratory failure, long-term oxygen therapy is recommended in the event of severe respiratory failure 
defined by $\mathrm{PaO}_{2} \leq 55 \mathrm{mmHg}(7.3 \mathrm{kPa})$, or $\mathrm{PaO}_{2}$ between 55 and $60 \mathrm{mmHg}(7.3-8.0 \mathrm{kPa})$ with at least one of the following criteria: polycythemia (hematocrit> 55\%), signs of pulmonary hypertension, and signs of right heart failure.

- Respiratory rehabilitation: a respiratory rehabilitation program must be discussed on a case-by-case basis.

- Despite the conflicting efficacy data, the use of intravenous cyclophosphamide remains the most widely used treatment for SScc-ILD. The schedule of administration every 4 weeks is that usually used. The dose is $0.7 \mathrm{~g} / \mathrm{m}^{2}$ or $0.5 \mathrm{~g} / \mathrm{m}^{2}$ in patients over 65 years of age or with GFR $<30 \mathrm{ml} / \mathrm{min} / \mathrm{m}^{2}$, at the rate of one treatment every 28 days for 12 months. The dose of cyclophosphamide is capped at $1200 \mathrm{mg} /$ injection. Intravenous uromitexan is administered concomitantly dose for dose. The total duration of 1 year of treatment is justified by the fact that, after a 6 -month treatment of intravenous cyclophosphamide followed up with PO azathioprine for 18 months, some initially responsive patients subsequently worsened ${ }^{13}$.

- MMF at a dose of $1500 \mathrm{mg}$, twice a day for 2 years has shown non-inferiority in a randomized study carried out versus oral cyclophosphamide and may be an alternative as a first-line treatment, especially for forms of ILD with a poorer prognosis ${ }^{14}$.

- Low-dose corticosteroid doses are recommended in association with cyclophosphamide or MMF. Given the risks of a renal crisis occurring in scleroderma patients, we recommend the use of corticosteroids at dosages $\leq 15 \mathrm{mg} /$ day of oral prednisone $\mathrm{e}^{15}$.

- RTX. For patients not responding to first-line therapies, consideration is given to RTX as rescue therapy. To assess the effect of RTX on the lung function parameters in SSc-ILD patients, PubMed and EMBASE were searched to identify studies on SScILD treated with RTX, confined to a predefined inclusion and exclusion criteria. A systematic review and meta-analysis were performed on the included studies on changes in forced vital capacity (FVC) and diffusion capacity of carbon monoxide (DLCO) from baseline to six and 12 months of follow-up. A total of 20 studies (two randomized controlled trials [RCT], six prospective studies, five retrospective studies, and seven conference abstracts) were analyzed. In conclusion, the treatment with RTX in SSc-ILD was associated with a significant improvement of both FVC and DLCO during the $1^{\text {st }}$ year of treatment. RTX use was associated with lower infectious adverse events ${ }^{16}$.
Table 2. Systemic sclerosis pulmonary hypertension treatment

\begin{tabular}{|c|c|}
\hline Functional class & Treatment \\
\hline NYHP & \\
\hline $\begin{array}{l}\text { Mild Functional Class I-II } \\
1^{\text {st }} \text { line } \\
2^{\text {nd }} \text { line } \\
3^{\text {rd }} \text { line }\end{array}$ & $\begin{array}{l}\text { PDE5i } \\
\text { ERA } \\
\text { Prostanoid }\end{array}$ \\
\hline $\begin{array}{l}\text { More severe functional Class III-IV } \\
1^{\text {st }} \text { line } \\
2^{\text {nd }} \text { line } \\
3^{\text {rd }} \text { line } \\
4^{\text {th }} \text { line }\end{array}$ & $\begin{array}{l}\text { Prostanoid } \\
\text { ERA + PDE5i } \\
\text { ERA + Prostanoid } \\
\text { Lung + Heart } \\
\text { Transplantation }\end{array}$ \\
\hline
\end{tabular}

NYHA: New York Heart Association; PDE5i: phosphodiesterase-5 inhibitor; ERA: endothelin receptor antagonist. Adapted from Fernández-Codina et al., 2018 ${ }^{19}$.

- Tocilizumab has been assessed in recent cutaneous forms with inflammatory profile. Both the Phase II and Phase III studies were negative for the primary criterion (Rodnan score). For one of the secondary criteria (pulmonary interstitial damage), there was a definite preservation of lung volumes $(\mathrm{FVC})^{17}$.

- Nintedanib, an oral triple-kinase inhibitor targeting pro-fibrotic pathways, has been used for the treatment of idiopathic pulmonary fibrosis. Based on positive results from a phase III, placebo-controlled, randomized comparative clinical trial conducted in patients with SSc-ILD, nintedanib received marketing approval for the treatment of SSc-ILD. Nintedanib significantly reduced the annual rate of decline in FVC over 52 weeks compared with placebo patients. The most common adverse events were gastrointestinal disorders, including diarrhea, nausea, and vomiting, which sometimes lead to discontinuation or permanent dose reduction of nintedanib. In contrast, serious adverse events were infrequent and were related mostly to worsening of cardiopulmonary involvement of SSc. Is used at the $150 \mathrm{mg}$ dose morning and evening; can be associated with immunosuppressants, especially MMF ${ }^{18}$.

\section{Pulmonary arterial hypertension}

In the treatment for $\mathrm{PAH}$, the sequential for mild and severe form of the disease was proposed (Table 2) ${ }^{19}$.

\section{Endothelin receptor antagonists}

Bosentan is an orally active mixed endothelin A and $B$ receptor antagonist approved for $\mathrm{PAH}$ associated 
with a connective tissue disease by the New York Heart Association (NYHA) functional Class II or III. Bosentan is started at a dosage of $62.5 \mathrm{mg}$ mornings and evenings for four weeks, then increased to a dosage of $125 \mathrm{mg}$ mornings and evenings according to hepatic tolerance (monthly hepatic testing obligatory [SGOT, SGPT] and routine monitoring of hemoglobin).

The benefit also provided by bosentan on the secondary prevention of digital ulcers can lead to recommending first-line use of bosentan in case of SScassociated $\mathrm{PAH}$ if the patient has a severe digital ulcer disease.

Ambrisentan is an orally active endothelin A receptor antagonist approved for $\mathrm{PAH}$ associated with connective tissue disease of NYHA functional Class II or III. The dosage is $5 \mathrm{mg}$ once per day and can be increased to $10 \mathrm{mg} /$ day.

\section{Phosphodiesterase-5 inhibitors}

Sildenafil and tadalafil are approved in the treatment of idiopathic, familial, or SSc-associated PAH with dyspnea of NYHA functional Class II or III. The dosage used is $20 \mathrm{mg} 3$ times/day for sildenafil and $2 \times 20 \mathrm{mg}$ once a day for tadalafil. There is no specific biological monitoring for these treatments.

\section{Continuous intravenous prostacyclin injection}

Epoprostenol is approved in the treatment of PAH associated with connective tissue disease of NYHA functional Class III or IV. It is administered in continuous intravenous perfusion through a portable Perfusor connected to a tunneled central venous catheter. In urgent situations, it can be administered through a peripheral venous route for a short duration while the central venous route is being set up. Epoprostenol constitutes the reference treatment for severe forms of SSc-associated PAH of functional classes III/IV.

\section{Prostacyclin receptor agonists}

Selexipag is a selective prostacyclin receptor agonist that is used orally. It is approved for treating PAH associated with connective tissue diseases of NYHA functional Class III and insufficiently controlled by treatment associating an endothelin receptor antagonist and a phosphodiesterase- 5 inhibitor. It is prescribed at a progressive dose over several weeks up to the maximum tolerated dose, to a maximum of $1600 \mu \mathrm{g}$ twice a day, based on tolerance. Special monitoring for the occurrence of adverse effects is crucial (headaches, flushing, and digestive disorders $)^{10}$

\section{Lung or heart-lung transplant}

This is the last recourse in case of severe PAH insufficiently improved by maximum medical treatment. The indication of transplant is systematically posed by reference centers or a competence center ${ }^{20}$.

\section{Treatment for scleroderma renal crisis (SRC)}

- Preventive treatment: prophylactic administration of angiotensin-converting enzyme (ACE) inhibitors has not yet been shown to be effective in preventing the occurrence of SRC. In contrast, treatment with prednisone at a dosage $>15 \mathrm{mg} /$ day within the previous three months appears to be associated with the occurrence of SRC. In this context, the prescription of corticosteroids should always be subject to expert advice

- Curative treatment: ACE inhibitors are the only therapeutic class to have demonstrated efficacy and to have modified the SRC prognosis. This demonstration is based on cohort follow-up studies. In the absence of hemodynamic instability, it is recommended the use of an intermediate half-life ACE inhibitor such as enalapril or ramipril in graduated doses. Captopril (short-acting ACE inhibitor) is only used in cases of hemodynamic instability. Conventional increases in creatinine levels with ACE inhibitors (lowering of renal perfusion pressure) should not result in a decrease in dosage ${ }^{2}$.

- Nicardipine or urapidil can be used early if blood pressure is not controlled by ACE inhibitors alone. The use of iloprost is recommended by some authors, but has not been validated. Use of bosentan cannot currently be recommended in the absence of clinical studies demonstrating its efficacy. At present, there is no demonstrated indication for first-line plasma exchange, eculizumab, or immunosuppressants. Corticosteroid therapy is contraindicated ${ }^{21}$.

\section{Treatment of digestive disorders}

\section{ESOPHAGITIS AND ESOPHAGEAL MOTOR DISORDERS}

- Hygienic-dietary measures, such as reduced sized meals, reducing or even stopping consumption of tobacco, alcohol, tea, coffee, and chocolate. 
Consultation with the nutrition team or a dietician is recommended.

- Postural rules.

- Anti-secretory therapy: double- and even quadruple-dose proton-pump inhibitors.

\section{GASTROPARESIS}

Dietary management is always necessary (fragmentation of meals, mixed diet). Low-residue diets and vitamin supplements have been recommended based on empirical evidence.

Stomach prokinetics accelerate gastric emptying, but can have a negative effect on motility of the small intestine when prescribed at too high a dose. Treatment with erythromycin is, therefore, recommended at a daily dosage not to exceed $125-250 \mathrm{mg} \times 2 /$ day. Concomitant use of erythromycin and colchicine is not recommended due to the potential for potentiation of colchicine side effects.

Should erythromycin fail, one can try amoxicillin/ clavulanic acid contained clavulanic acid which is prokinetic for the stomach.

Prokinetic treatment with metoclopramide or metopimazine may be proposed if there are no neurological and/or electrocardiographic contraindications.

Gastroparesis can lead to a state of severe undernutrition, requiring prolonged enteral (jejunal) feeding.

\section{INTESTINAL DISORDERS}

1. Motor disturbances causing malabsorption syndrome and/or pseudo-obstruction of the intestine: in the case of acute occlusion, the first-line treatment consists of rehydration, analgesia (preferably avoiding opioids, which tend to exacerbate intestinal dysmotility), and relief of the small intestine by nasogastric aspiration. Nutritional management should not be delayed. Intestinal prokinetic agents can be used. Metoclopramide and domperidone often have little efficacy. The action of erythromycin on the motility of the small intestinal is less well known. Intravenous neostigmine can be used in acute episodes, but cardiac and cholinomimetic adverse events limit its use in frail patients.

2. Malabsorption syndrome by chronic bacterial colonization of the small intestine: the treatment is based on monthly sequential oral antibiotic therapy with alternating courses of different treatments, or even periods without treatment. The alternation of antibiotic molecules is proposed to avoid the emergence of a multiresistant intestinal bacterial flora. The durations are 10-14 days/month, and an alternation of three antibiotic molecules from different families is usually proposed. Commonly used antibiotics are amoxicillin (500 mg $\times 3 /$ day) or ciprofloxacin (250 $\mathrm{mg} \times 2 /$ day) or other quinolones, doxycycline (100 mg/day) (and other tetracyclines), metronidazole (250 mg $\times 3 /$ day), gentamicin (80 mg/day) or neomycin (500 mg $\times 4 /$ day), and sulfamethoxazole 800 mg-trimethoprim $160 \mathrm{mg}$ ( 1 tab $2 \times$ per day).

3.Colonic disease: the treatment of constipation is based on hygiene-dietary measures (balanced diet of fiber and mucilage, satisfactory hydration, and regular physical activity), laxatives, and evacuating enemas. The advice of the nutrition team and/or a dietician is recommended for severe forms. Prokinetic drugs can be combined to improve colon motility (and to a lesser extent symptoms): metoclopramide 20-30 mg daily, domperidone (the maximum daily dose is currently $30 \mathrm{mg}$ daily in three doses), and prucalopride once daily $(2 \mathrm{mg}$ before the age of 65 and $1 \mathrm{mg}$ over). Their use must be limited in time ${ }^{22}$.

\section{Future and investigative treatment}

A recent study carried out in mice in which an experimental SSc is induced, investigated the effect of $B$ cell depletion on fibrosis in systemic sclerosis (SSc) and its mechanism of action. Mice with bleomycin-induced SSc (BLM-SSc) were treated with anti-CD20 antibody, and skin and lung fibrosis were histopathologically evaluated. $T$ cells and macrophages were co-cultured with $B$ cells, and the effect of $B$ cells on their differentiation was assessed by flow cytometry. B-cell depletion inhibited fibrosis in mice with BLM-SSc. B cells from mice with BLM-SSc increased pro-inflammatory cytokine-producing T cells in co-culture. In mice with BLMSSc, B-cell depletion before BLM treatment (pre-depletion) inhibited fibrosis more strongly than B-cell depletion after BLM treatment (post-depletion) $(p<0.01)$. However, the frequencies of pro-inflammatory $T$ cells were lower in the post-depletion group than in the pre-depletion group. This discrepancy suggests that the effect of B-cell depletion on fibrosis cannot be explained by its effect on T-cell differentiation. On the other hand, profibrotic macrophages were markedly decreased in the pre-depletion group compared to the post-depletion group $(p<0.05)$. Furthermore, B cells from mice with BLM-SSc increased profibrotic 
macrophage differentiation in coculture $(p<0.05)$. In SSc patients, the extent of profibrotic macrophage induction by $\mathrm{B}$ cells correlated with the severity of fibrosis $(p<0.0005)$. In conclusion: these findings suggest that B-cell depletion inhibits tissue fibrosis through suppression of profibrotic macrophage differentiation in mice with BLM-SSc, providing a new rationale for B-cell depletion therapy in $\mathrm{SSc}^{23}$.

Chimeric antigen receptor-T (CAR-T) cell therapy is based on the specific targeting of tumor antigens, leading to lysis and the destruction of tumor cells. The high potency of CAR-T cells in the management of B-cell malignant neoplasms has been demonstrated. Following the success of this therapeutic strategy, new constructs derived from CAR-T cells have been developed that can eradicate pathogen $\mathrm{B}$ cells or restore tolerance.

The knowledge and technology generated using CAR-T cells can be translated and integrated into ongoing therapeutic strategies for autoimmune rheumatic diseases, such as rheumatoid arthritis, generalized lupus erythematosus, and $\mathrm{SSc}^{24}$.

To assess the preclinical efficacy and mechanism of action of an anti-CX ${ }_{3} \mathrm{CL} 1$ monoclonal antibody $(\mathrm{mAb})$ in systemic sclerosis (SSc), cultured human dermal fibroblasts were used to evaluate the direct effect of anti$\mathrm{CX}_{3} \mathrm{CL} 1 \mathrm{mAb}$ on fibroblasts. Anti-CX ${ }_{3} \mathrm{CL} 1 \mathrm{mAb}$ treatment significantly inhibited Smad3 phosphorylation $(p<0.05)$ and expression of type I collagen and fibronectin 1 $(p<0.01)$ in dermal fibroblasts stimulated with transforming growth factor $\beta 1$ (TGF $\beta 1$ ). In the bleomycin model, daily subcutaneous bleomycin injection increased serum $\mathrm{CX}_{3} \mathrm{CL1}$ levels $(\mathrm{p}<0.05)$ and augmented lesional $\mathrm{CX}_{3} \mathrm{CL} 1$ expression. Simultaneous administration of anti-CX ${ }_{3} \mathrm{CL} 1 \mathrm{mAb}$ or $\mathrm{CX}_{3} \mathrm{CR} 1$ deficiency significantly suppressed the dermal thickness, collagen content, and capillary loss caused by bleomycin $(p<0.05)$. Injection of bleomycin-induced expression of pSmad3 and TGF $\beta 1$ in the skin, which was inhibited by anti-CX ${ }_{3} \mathrm{CL} 1 \mathrm{mAb}$. Further, the dermal infiltration of $\mathrm{CX}_{3} \mathrm{CR} 1+$ cells, macrophages (inflammatory and alternatively activated [M2-like] subsets), and CD3+ cells significantly decreased following anti-CX ${ }_{3} \mathrm{CL} 1$ mAb therapy $(p<0.05)$, as did the enhanced skin expression of fibrogenic molecules, such as thymic stromal lymphopoietin and secreted phosphoprotein 1 $(p<0.05)$. However, the treatment did not significantly reduce established skin fibrosis. In the second model, simultaneous anti-mCX ${ }_{3} \mathrm{CL} 1 \mathrm{mAb}$ therapy significantly diminished the skin fibrosis induced by serial subcutaneous injection of TGF $\beta$ and connective tissue growth factor $(p<0.01)$. In conclusion, this study suggests that anti-CX ${ }_{3} \mathrm{CL} 1 \mathrm{mAb}$ therapy may be a novel approach for treating early skin fibrosis in inflammation-driven fibrotic skin disorders such as $\mathrm{SSc}^{25}$.

A Phase II study, carried out in the USA, studied the efficacy of abatacept in SSc with diffuse skin involvement, in an early phase. It was a multicenter, double-blind, randomized, placebo-controlled study; however, the results did not allow us to establish changes between the groups of statistical significance ${ }^{26}$

A meta-analysis carried out in Portugal assessed the use of botulinum toxin to treat peripheral vascular disease, which includes RP and digital ulcers. The authors conducted a review of the literature on the subject and identified 30 results, of which five original papers were included: two RCTs, two case series, and one casecontrol study, from a total 133 patients. Only one RCT showed negative results, with worse blood flow in the treated arm, but with lower dose of botulinum toxin. Despite this, all five included studies reported improvement of a least one RF/hand function outcome measure. Concerning digital ulcer healing, resolution of baseline digital ulcer at the end of follow-up was reported in $75-100 \%$ of the patients, with one RCT showing superiority over placebo. Botulin toxin protocols were highly heterogeneous. In conclusion, botulin toxin is a valid option in the treatment of SSc-related peripheral vasculopathy. However, future large prospective trials are necessary ${ }^{27}$.

The autologous hematopoietic stem cell transplantation (HSCT) allowed a rapid and sustained improvement in skin and lung fibrosis, proved for early phase I/II studies, along with improved long-term survival of up to seven years after HSCT. The Canadian Scleroderma Research Group has done a recent study to quantify the magnitude, domains, and duration of change in health-related quality of life (HRQoL) in patients with systemic sclerosis (SSc) who underwent autologous HSCT as compared to SSc patients with similar characteristics who did not undergo autologous HSCT. In total, 41 SSc patients who underwent autologous HSCT and 65 SSc patients treated with conventional care were compared. This study provides robust complementary HRQoL data, including overall and event-free survival data, to expand on the standard repertoire of biomedical variables, thus potentially supporting the physical benefits of autologous HSCT in patients with $\mathrm{SSc}^{28,29}$. 


\section{Funding}

None.

\section{Conflicts of interest}

None.

\section{Ethical disclosures}

Protection of human and animal subjects. The authors declare that no experiments were performed on humans or animals for this study.

Confidentiality of data. The authors declare that no patient data appear in this article.

Right to privacy and informed consent. The authors declare that no patient data appear in this article.

\section{References}

1. Yen EY, Sing DR, Sing RR. Trends in systemic sclerosis mortality over forty-eight years,1968-2015: a US population-based study. Arthritis Care Res (Hoboken). 2021;73:1502-10.

2. Hickman RJ. ACE inhibitors in scleroderma renal crisis. Reumatologist. 2021;15:7-8.

3. di Martina O, Lepri G, Damiani A, Barsotti S, Di Batista M, Codullo V One year in review 2020: systemic sclerosis. Clin Exp Rheumatol. 2020;38 Suppl 125:3-17.

4. Sullivan KM, Majhail NS, Bredeson C, Carpenter PA, Chatterjee S, Crofford LJ, et al. Systemic sclerosis as an indication for autologous hematopoietic cell transplantation; position statement from the American society for blood and marrow transplantation. Biol Blood Marrow Transplant. 2018;241961-4

5. Rirash F, Tingey PC, Harding SE, Maxwell LJ, Ghogomou ET, Wells GA et al. Calcium channel blockers for primary and secondary Raynaud's phenomenon. Cochrane Database Syst Rev. 2017;12:CD00467.

6. Pope J, Fenlon D, Thompson A, Shea B, Furst D, Wells GA, et al. Iloprostot and cisaprost for Raynaud's phenomenon in progressive systemic sclerosis. Cochrane Database Syst Rev. 2000;1998:CD000953.

7. Hinchcliff M, Varga J. Systemic sclerosis/scleroderma: a treatable multisystem disease. Am Fam Physician. 2008;78:961-8.

8. Kowal-Bielecka O, Fransen O, Avouac J, Becker M, Kulac A, Allanore Y, et al. Update of EULAR recomendations for the treatments of systemic sclerosis. Ann Rheum Dis. 2017;76:1327-39.

9. Matucci-Cerinic M, Denton CP, Furst DE, Mayes MD, Hsu VM Carpentier $\mathrm{P}$, et al. Bosentan treatment of digital ulcers related to systemic sclerosis: result FROM RAPIDS-2 randomised, double-blind, placebo- controlled trial. Ann Rheum Dis. 2011;70:32-8.
10. Hachulla E, Agard C, Allanore Y, Avouac J, Bader-Meunier B, Belot A, et al. French recommendations for the management of systemic sclerosis. Orphanet J Rare Dis. 2021;16 Suppl 2:322.

11. Khana D, Clements PJ, Volkmann ER, Wilhalme $\mathrm{H}$, Tseng $\mathrm{CH}$, Furst $\mathrm{DE}$, et al. Minimal clinically important differences for the modified Rodnan skin score: results from the scleroderma lung studies (SLS-I and SLS-II). Arthritis Res Ther. 2019;21:23.

12. Young A, Namas R, Dodge C, Khanna D. Hand impairment in systemic sclerosis: various manifestations and currently available treatment. Curr Treatm Opt Rheumatol. 2016;2:252-69.

13. Fischer A, Patel NM, Volkmann ER. Interstitial lung disease in systemic sclerosis: focus on early detection and intervention. Open Access Rheumatol. 2019;11:283-307.

14. Cassone G, Sebastiani M, Vacchi C, Erre GL, Salvarani C, Manfredi A Efficacy and safety of mycophenolate mofetil in the treatment of rheumatic disease-related interstitial lung disease: a narrative review. Drugs Context. 2021;10:2020-8-8.

15. Iudici M, van der Goes MC, Valentini G, Bijlsma JW. Glucocorticoids in systemic sclerosis: weighing the benefits and risks-a systematic review. Clin Exp Rheumatol. 2013;31 Suppl 76:157-65.

16. Goswami RP, Ray A, Chatterjee M, Mukherjee M, Sircar G, Ghosh P. Rituximab in the treatment of systemic sclerosis-related interstitial lung disease: a systematic review and meta-analysis. Rheumatology (Oxford). 2021;60:557-67

17. Khanna D, Lin CJ, Furst DE, Goldin J, Kim G, Kuwana M. Tocilizumab in systemic sclerosis: a randomised, double-blind, placebo-controlled, phase 3 trial. Lancet Respir Med. 2020;8:963-74.

18. Kuwana M, Azuma A. Nintedanib: new indication for systemic sclerosis-associated interstitial lung disease. Mod Rheumatol. 2020;30:225-31.

19. Fernández-Codina A, Walker KM, Pope JE, Scleroderma Algorithm Group. Treatment algorithms for systemic sclerosis according to experts. Arthritis Rheumatol. 2018;70:1820-8.

20. Naranjo M, Hassoun PM. Systemic sclerosis-associated pulmonary hypertension: spectrum and impact. Diagnostics (Basel). 2021;11:911.

21. Nagaraja V. Management of scleroderma renal crisis. Curr Opin Rheumatol. 2019;31:223-30

22. Gyger G, Baron M. Systemic sclerosis: gastrointestinal disease and its management. Rheum Dis Clin North Am. 2015;41:459-73.

23. Numajiri H, Kuzumi A, Fukasawa T, Ebata S, Yoshizaki-Ogawa A, Asano Y. Arthritis Rheumatol. 2021;73(11):2086-95

24. Boulch M, Bousso P, Allanore $Y$, Avouac J. Is there a place for chimeric antigen receptor-T cells in the treatment of chronic autoimmune rheumatic diseases? Arthritis Rheumatol. 2021;73:1954-65.

25. Luong VH, Utsunomiya A, Chino T, Doanh LH, Matsushita T, Obara T, et al. Inhibition of the progression of skin inflammation, fibrosis, and vascular injury by blockade of the cx3c11/cx3cr1 pathway in experimental mouse models of systemic sclerosis. Arthritis Rheumatol. 2019;71:1923-34.

26. Khanna D, Spino C, Johnson S, Chung L, Whitfield ML, Denton CP, et al. Abatacept in early diffuse cutaneous systemic sclerosis: results of a phase II investigator-initiated, multicenter, double-blind, randomized, placebo-controlled trial. Arthritis Rheumatol. 2020;72:125-36.

27. Guerra MG, da Fonseca DG, Samôes B, Videira T, Pinto P. Is Botulinum Toxin Useful in Systemic Sclerosis Related Peripheral Vasculopathy? A Literature Review.. Reumatol Clin. 2021;17:357-63.

28. Maltez N, Puyade M, Wang M, Lansiaux P, Marjanovic Z, Charles C, et al. Association of autologous hematopoietic stem cell transplantation in systemic sclerosis with marked improvement in health-related quality of life. Arthritis Rheumatol. 2021;73:305-14.

29. Sullivan KM, Goldmuntz EA, Keyes-Elstein L, McSweeney PA, Pinckney A Welch B. Myeloablative autologous stem-cell transplantation for severe scleroderma. N Engl J Med. 2018;378:35-47. 